Relations industrielles

Industrial Relations

\title{
Jean-Louis Dubé, Décrets et comités paritaires
}

\section{Fernand Morin}

Volume 46, numéro 1, 1991

URI : https://id.erudit.org/iderudit/050661ar

DOI : https://doi.org/10.7202/050661ar

Aller au sommaire du numéro

\section{Éditeur(s)}

Département des relations industrielles de l'Université Laval

\section{ISSN}

0034-379X (imprimé)

1703-8138 (numérique)

Découvrir la revue

Citer ce compte rendu

Morin, F. (1991). Compte rendu de [Jean-Louis Dubé, Décrets et comités paritaires]. Relations industrielles / Industrial Relations, 46(1), 240-241.

https://doi.org/10.7202/050661ar

Tous droits réservés (C) Département des relations industrielles de l'Université Laval, 1991
Ce document est protégé par la loi sur le droit d'auteur. L’utilisation des services d'Érudit (y compris la reproduction) est assujettie à sa politique d'utilisation que vous pouvez consulter en ligne.

https://apropos.erudit.org/fr/usagers/politique-dutilisation/ 
Décrets et comités paritaires, par Jean-Louis DuBE, les éditions Revue de droit, Université de Sherbrooke, 1990, 376 p., ISBN 2-92003-13-5

Le professeur Jean-Louis Dubé nous offre cette année une étude de l'application de la Loi sur les décrets de convention collective. L'ouvrage comprend trois parties principales et toutes nécessaires pour saisir la portée juridique et pratique de cette loi: titre I: le champ d'application; titre II: le processus d'extension d'une convention collective et titre III: les effets d'un décret. On y ajoute, en amont et en aval, un rappel de la genèse de cette loi de 1934 et la vision de l'auteur au sujet de sa fonction future. Dès l'introduction, monsieur Dubé souligne l'objet et la finalité de la loi de manière à ce que tout lecteur sache bien de quoi il s'agit: assujettir employeurs et salariés d'un même marché à une convention collective conclue par un certain groupe d'entre eux. La genèse de cette loi conserve un grand intérêt historique car elle témoigne d'une double source d'influence: l'approche européenne pour aborder les questions des relations du travail et la doctrine sociale de l'Église catholique bien connue à cette époque.

Le premier titre traite des personnes et des aires professionnelles et territoriales. Bien que la définition du salarié retenue soit fort différente de celle du Code du travail, le professeur Dubé constate que l'on exige néanmoins un lien de subordination juridique (p. 48). S'il est vrai que les libellés ne partagent aucun élément commun en ces deux définitions, il est aussi exact que la définition donnée au Code du travail ne traite nullement de cette même subordination ou assujettissement et pourtant, on l'a toujours imposée, depuis 1944. N'est-ce pas parce que l'approche civiliste transcende en toute interprétation judiciaire des lois du travail? L'auteur s'interroge à savoir s'il s'agit véritablement d'une loi du travail ou de l'encadrement d'activités économiques et commerciales (p. 50). Cette même question est reprise afin de déterminer s'il est possible, par la voie d'un décret, d'imposer un prix pour certains services (cordonnerie, coiffure, etc.) ou encore, pour établir les heures d'ouverture des commerces, etc. (p. 155). Par la suite, l'auteur nous fournit une démonstration convaincante de la difficile tâche d'arrêter des lignes frontalières permettant de circonscrire les aires professionnelles d'un décret particulier et son territoire propre. Un tel exercice est toujours laborieux, mais il le serait davantage lorsque l'on tente de cerner des activités qui évoluent en fonction de méthodes ou de technologies en constant renouvellement et surtout, lorsqu'il n'existe pas de critères ni de procédures préalablement arrêtés pour effectuer ce travail. Pour ces raisons, sans doute, l'auteur préfère se limiter à décrire ce qui se fait à l'aide d'exemples (p. 69-77 et p. 97-105). D'une façon assez surprenante, le professeur Dubé propose que le Tribunal du travail tranche ces conflits des aires d'application des décrets (p. 96) et suggère aussi d'utiliser les régions administratives pour établir les «zones de salaire» (p. 108). Cette double proposition est, tout le moins, prématurée car il faudrait d'abord que le législateur intervienne sur le fonds même de la loi et la mettre à jour avant de choisir l'arbitre approprié. La détermination des régions administratives répondaient à de nombreuses considérations, nous semble-t-il, mais qui sont assez éloignées des préoccupations particulières à chaque décret.

Le titre II porte sur l'adoption du décret soit la convention collective lui servant de noyau, la procédure publique d'extension et son contenu légalement possible (p. 109-177). Bien que la convention collective conclue en vertu du Code du travail puisse aussi servir à cette fonction, il n'est nullement nécessaire qu'il en soit ainsi. La Loi sur les décrets de convention collective ne dit mot quant au processus de négociation et monsieur Dubé souligne que la convention peut être conclue sous condition suspensive à l'arrivée d'un décret (p. 129). L'important consisterait à ce que les sujets, tant les salariés que les employeurs, soient suffisamment représentatifs pour conférer à leur convention collective «... une signification importante prépondérante» justifiant de ce fait son extension (p. 133). Il est bien évident que ces silences à la loi quant aux critères de qualification des parties signataires et de la convention collective confèrent une très 
grande discrétion au ministre responsable. Pour pondérer justement cette discrétion administrative, l'auteur propose, à l'instar de l'industrie du bâtiment, que les modifications d'autorité apportées à un décret soient précédées: «... d'une audition des parties en Commission parlementaire» (p. 149). L'importance du décret de la construction pouvait peut-être expliquer l'usage d'un tel moyen extraordinaire, mais de là à le banaliser pour tout décret, il y a une marge difficile à jauger.

Le titre III traite du caractère impératif du décret dans le sens que les conditions de travail ainsi imposées ne peuvent être réduites ni contournées par un contrat ou une convention collective du travail. Par ailleurs, les conditions de travail objectivement plus avantageuses peuvent être obtenues à l'une ou l'autre de ces deux voies. L'auteur rappelle plusieurs décisions judiciaires qui ont clarifié ces questions:

- un employeur lié à une convention collective selon le Code du travail demeure néanmoins soumis à un décret, s'il y a lieu;

- un employeur soumis à un décret peut être contraint à négocier avec le syndicat accrédité de ses salariés.

Le professeur Dubé souligne que l'administration du décret est confiée non pas à un organisme étatique, comme c'est le cas en plusieurs pays européens, mais à un comité paritaire formé de représentants des syndicats et des employeurs visés.

Au dernier titre, l'auteur propose un certain nombre de réformes dans le but de tirer profit des expériences acquises au cours des cinquante ans d'application de cette loi. À cette occasion, monsieur Dubé touche le point le plus nébuleux, soit les caractéristiques objectives d'une convention collective pouvant faire l'objet d'une extension juridique (p. $267 \mathrm{et} \mathrm{ss}$.). Il nous propose l'institution d'une commission de l'extension juridique (p. 269) qui effectuerait le travail d'évaluation de la qualité de la convention collective et des parties visées, sans toutefois disposer d'un pouvoir décisionnel. En d'autres termes, ce travail de tamisage ne réduirait pas la discrétion administrative du ministre. Cette opération préalable, coûteuse et sans doute dilatoire, épargnerait seulement le ministre du poids des revendications ouvertes, sans plus. Le professeur Dubé suggère également que le comité paritaire soit présidé par un représentant du ministère (p. 274). Il nous est encore difficile de saisir la justification de ces deux propositions qui consistent à imposer une tutelle étatique. Ne serait-ce pas le meilleur moyen pour que les parties évitent l'usage de cette troisième voie? De plus, on ne saurait ainsi les inciter à assumer vraiment leur réelle et mutuelle responsabilité. La question est importante car le régime actuel retenu au Code du travail (une entreprise ou partie d'entreprise à la fois) répond de moins en moins aux besoins actuels notamment dans le secteur tertiaire où $70 \%$ des emplois s'y retrouvent.

L'ouvrage du professeur Dubé renferme une source d'information fort valable. On rend compte de l'approche des tribunaux, des difficultés pratiques de l'administration d'un tel système tant de la part de l'État que de celle des parties et des multiples études ou recherches entreprises pour en corriger le tir. Il se peut que l'auteur ait raison en affirmant que cette loi pourrait servir de troisième voie à l'élaboration des conditions de travail entre la négociation atomiste du Code du travail et l'intervention directe de l'État en vertu de la Loi sur les normes du travail. Cette seule possibilité justifie amplement de mieux connaître les tenants et aboutissants de cette Loi sur les décrets de convention collective, ce que nous permet et facilite ce volume.

Fernand MORIN 